\title{
A laboratory of polarization in a Master degree of photonics
}

\section{J. Campos, A. Lizana, O. Lopez, M. Yzuel}

J. Campos, A. Lizana, O. Lopez, M. J. Yzuel, "A laboratory of polarization in a Master degree of photonics," Proc. SPIE 9666, 11th Education and Training in Optics and Photonics Conference, 966612 (5 June 2009); doi: $10.1117 / 12.2207933$

SPIE Event: Eleventh International Topical Meeting on Education and Training in Optics and Photonics, 2009, St. Asaph, United Kingdom 


\title{
A laboratory of polarization in a Master degree of Photonics
}

\author{
Campos J., Lizana A., Lopez O. and Yzuel M.J. \\ Departamento de Física, Universidad Autónoma de Barcelona, 08193 Bellaterra, Spain. \\ maria.yzuel@uab.es
}

\begin{abstract}
Polarimetric techniques are widespread employed in many research fields as optics, medicine or biology. In this sense, the use of polarimeters has significantly increased, being a tool with huge perspectives of future. As a consequence, the spreading of the basic knowledge of this topic becomes interesting for many professionals and a master studies is an excellent environment to this aim.

We are participating in a mandatory laboratory subject (Laboratory of Optics, LO) of a Master degree in Photonics with an experiment on polarization. In particular, the main structure of the experiment has been built around of a polarimeter set-up. Basically, we use a He-Ne laser beam, a polarization state generator and a polarization state detector. The experimental measurements are acquired by means of a photometer connected to a computer and processed by an own developed software. It allows us to obtain a complete description of any polarizing element tested. In combination with the laboratory work, it is provided a mathematical description of the polarization theory, the Stokes-Mueller formalism, which gives us the base required for a fully understand of the experiment.

Throughout this work, we explain the polarimeter experiment structure and the achievements reached by students. We want to emphasize that a different degree of expertise and knowledge in function of the specific background of every student is provided. However, a minimum knowledge level is reached for all students, including among others, the improvement in the scientific, communicative or interdisciplinary competences.
\end{abstract}

Key words: Laboratory of Optics, polarimetry, polarimeter, Stokes, Mueller, synchronous detection.

\section{INTRODUCTION}

The knowledge of the state of polarization of light beams and of the polarimetric properties of polarizing samples is necessary in a large number of applications as in medical physics ${ }^{1,2}$, in astronomy ${ }^{3}$ or in polarizing samples characterization ${ }^{4,5}$, among others. In order to obtain this type of information, the use of polarimeters is required in these applications. Polarimeters are optical instruments capable to characterize the state of polarization of a light beam just by means of radiometric measurements. The radiometric data are obtained by projecting the analyzed light beam upon different configurations of a polarization state detector (different polarization analyzers). On the other hand, by properly combining a polarization state generator with $a$ polarization state detector, all the polarizing properties of polarizing samples can also be determined.

By taking into account the polarimetric data provided by polarimeters or its set-up design, they can be subdivided in different subtypes ${ }^{6}$. For instance, we can divide them as complete or incomplete (complete if fully determine light beams or polarizing samples, being incomplete otherwise), as light-measuring and sample-measuring polarimeters (light beam or polarizing sample characterization) or as a function of its data acquisition methodology.

In order to spread the basic knowledge related to polarimetry ${ }^{7}$, the Image Processing Laboratory (IPL) of the Optics Group of the University Autonomus of Barcelona (UAB) is participating in a mandatory laboratory subject (Laboratory of Optics, LO) of a Master degree in Photonics with an experiment on polarization. In particular, the main structure of the experiment has been built around of a complete polarimeter set-up. 
Basically, it uses a He-Ne laser beam in which a polarization state generator (polarizer and quarterwaveplate) and a polarization state detector (quarter-waveplate and analyzer) are inserted. The experimental data are acquired by means of a photometer connected to a computer and processed by an own developed LabVIEW software, leading to a complete description of any polarizing element tested.

The educational structure of the Laboratory of Optics subject is divided in three different units. In this sense, in the first unit the basis of the Mueller-Stokes (M-S) formalism is provided. The M-S formalism is a useful mathematical tool that allows the description of the state of polarization of light beams and of the interaction of light beams with polarizing samples. Note that this first unit is essential for the fully understanding of the experiment. Next, in the second section, the experimental calibration of the optical elements of the set-up is conducted. This is a first step for the assimilation of the theoretical concepts revised in the previous unit because the calibration protocol is based on the M-S formalism. Moreover, the calibration procedure taught in this unit is useful not only for the specific set-up used for the complete polarimeter implementation but also, in a more general way, for the optimization of optical systems based on polarizing elements. Finally, in the third unit of the LO, the polarimeter is used to characterize some samples. First, by using the detector system the students detect different types of polarized light. Then, by means of the fully operation of the polarimeter, the Mueller matrices of diverse polarizing samples are obtained: polarizer, waveplate, Faraday rotator, among others. Note that this unit is a key issue in the experiment, allowing for instance, the experimental manipulation of the set-up, the complementation of the theory with different experimental examples, and the polarimetric analysis of the obtained results. In addition to the experiment on polarimetry conducted by students, they have to deliver a mandatory report and to perform an oral presentation. These two works are very important for the experiment complete understanding. In this sense, the students achieve a further internalization of the experiment conducted, observe the benefits of the team work and improve their communicative skills.

The outline of this paper is as follows. In section 2, the Mueller-Stokes formalism basis explained to students is provided. In section 3, the calibration protocol followed by the students for the calibration of the optical elements of the set-up used is detailed. Next, in section 4 we show the characterization process used for the obtaining of the Stokes vectors of light beams and the Mueller matrices of polarizing samples. Finally, in section 5 a summary of the experiment and achievements reached by students is provided

\section{MUELLER-STOKES FORMALISM: MUELLER MATRICES OF THE MAIN OPTICAL ELEMENTS}

There exist different mathematical formalisms, as the Berreman or the Jones formalisms, which allow the correctly describing of the state of polarization (SoP) of light beams and the interaction of light beams with polarizing elements. However, in the cases where it is important to take into account unpolarized light contributions or depolarizing effects, the so-called Mueller-Stokes (M-S) formalism is very suitable and thus, it is used in a large number of applications. In the M-S formalism, the state of polarization of light beams is described by means of four real parameters that can be obtained readily by radiometric measurements and they are usually presented as a column vector (the Stokes vector). On the other hand, the description of polarizing samples is given by Mueller matrices, $4 \times 4$ matrices of real elements which keep useful polarimetric information. In addition, the Mueller matrices relate the incident and exiting (reflected, transmitted or scattered) states of polarization $\mathrm{S}$ of a light beam as follows:

$$
S_{\text {exiting }}=M \cdot S_{\text {incident }}
$$

where $\mathrm{M}$ is the Mueller matrix of a polarizing sample.

In the first unit of the experiment and with the aim of provide the students of the Master degree of Photonics with this useful and potent tool, the physical interpretation and the manipulation of the M-S formalism is taught. We want to emphasize that the degree of detail given to students strongly depends of the specific polarimetric background of every student. In this sense, for students without previous knowledge in this issue, 
an initiation to the M-S terminology and basic manipulation is provided. On the other hand, more specialized students are trained on more difficult problems related to polarimetry and we give them a further insight on this topic.

A basic knowledge that students have to learn when conducting this experiment is the awareness of the Mueller matrices of the main polarizing optical elements, as polarizers or waveplates. This is an important issue because the internalization of this information is the basis required for the achievement of more complex polarimetric problems. An example of different Mueller matrices representing typical optical elements is given in Table 1. Note that the Eq. (6) is very important when working with the M-S formalism because it leads to the obtaining of the Mueller matrix of any polarizing element rotated an angle $\theta$.

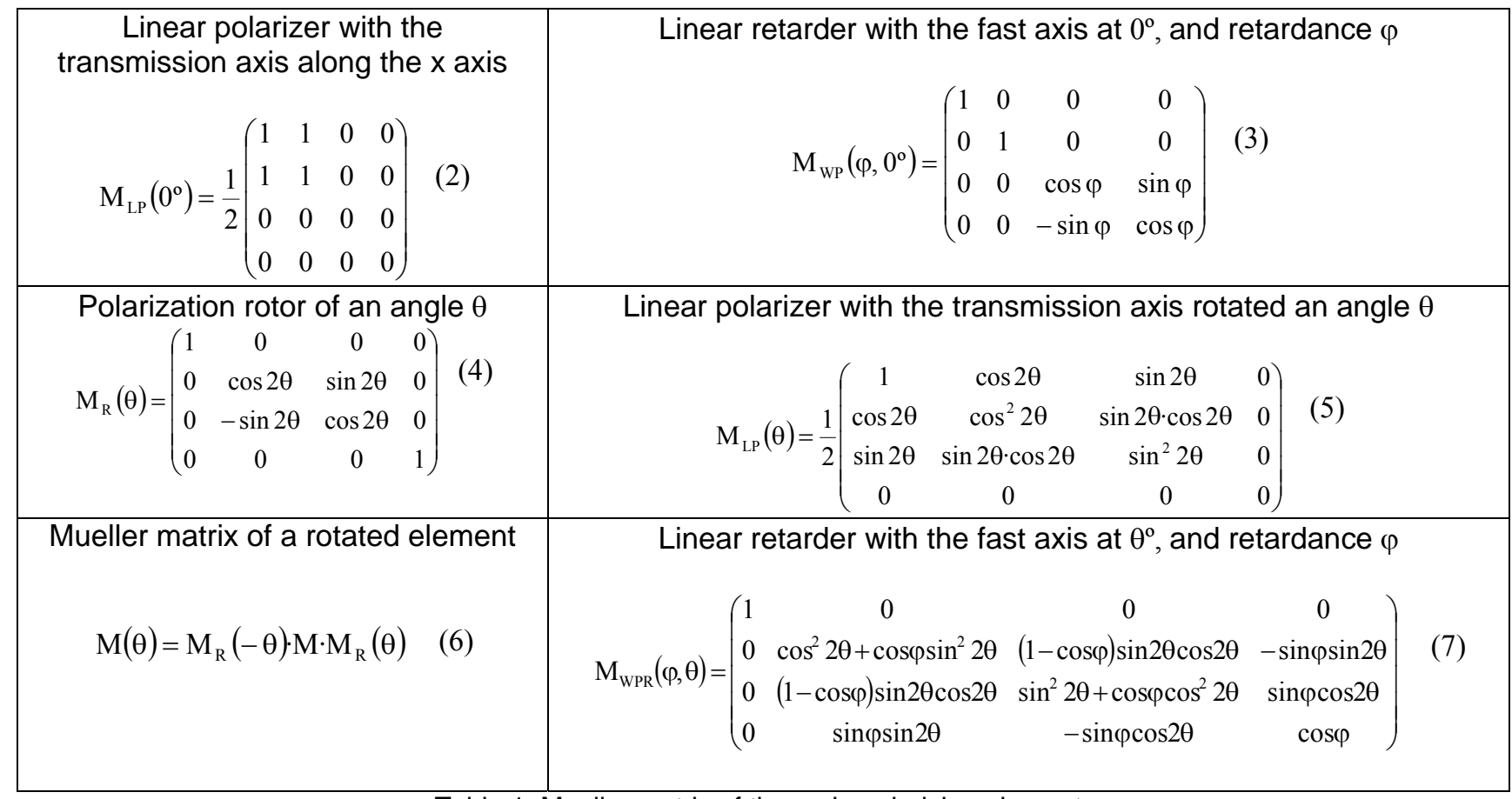

Table 1. Mueller matrix of the main polarizing elements.

\section{SET-UP CALIBRATION}

Once some fundamental theory related to polarimetry has been provided to the students, we begin with the second and third units of the experiment, where by means of an experimental set-up the students reinforce and consolidate the theoretical content. In this section, we detail the unit 2 , where the students perform the calibration of the experimental set-up. The main structure of the experiment has been built around of a polarimeter set-up and its corresponding design is plotted in Fig. 1. It contains a He-Ne laser beam, a polarization state generator (polarizer $\mathrm{LP}_{1}$ and waveplate $\mathrm{WP}_{1}$ ) and a polarization state detector (waveplate $\mathrm{WP}_{2}$ and analyzer $\mathrm{LP}_{2}$ ). The sample to be analyzed is placed between both. The waveplates can rotate 360 degrees and they are electronically controlled from a personal computer. The intensity measurements are acquired by means of a photometer connected to a Digital-to-Analog Converter (DAC) that sends the digital signals to a computer. Then, the set-up is controlled and the data is processed by an own developed LabVIEW software that the student may change. 


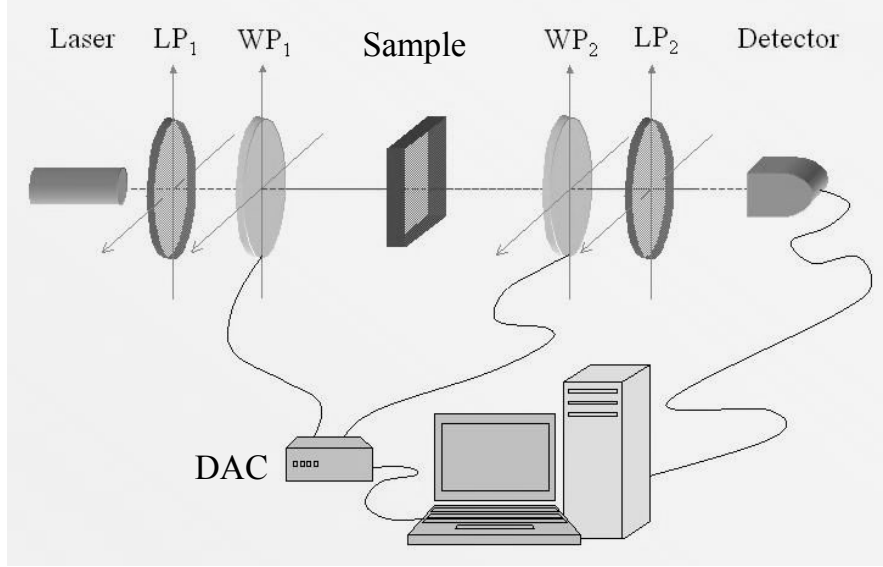

Figure 1. Polarimeter set-up.

The specific function of the different elements used in the set-up sketched in Fig. 1 is explained to the students. Then, we lead them into a set-up calibration protocol based on the synchronous detection ${ }^{8}$. We think that this knowledge is very useful because it is necessary not only to decrease the associated measurements errors when using the polarimeter but also to optimize the performance of any optical system involving polarizing elements.

\subsection{Determination of the rotation angle between two polarizers}

The first step for the characterization of the elements of our setup is the determination of the polarizer's orientation. Polarizers are mounted in manually rotary stages, graduated every two degrees, but the transmission axis may not coincide exactly with the zero of the rule. Then it is necessary to determine the position of the transmission axis. The first polarizer transmission axis is taken as our $\mathrm{x}$ axis, and all the elements are aligned with it. Then, after the first polarizer the Stokes vector is given by

$$
\left(\begin{array}{l}
\mathrm{S}_{0} \\
\mathrm{~S}_{1} \\
\mathrm{~S}_{2} \\
\mathrm{~S}_{3}
\end{array}\right)=\mathrm{t}\left(\begin{array}{l}
1 \\
1 \\
0 \\
0
\end{array}\right)
$$

Let us assume that the transmission axis of the second polarizer is at an unknown angle $\theta_{0}$ with respect to the zero of its rotary stage. Then when the rule of the rotary stage marks $\theta$, in fact the transmission axis is at $\theta+\theta_{0}$. Therefore, the Stokes vector after the second polarizer is

$$
\left(\begin{array}{l}
\mathrm{S}_{0}^{\prime} \\
\mathrm{S}_{1}^{\prime} \\
\mathrm{S}_{2}^{\prime} \\
\mathrm{S}_{3}^{\prime}
\end{array}\right)=\frac{\mathrm{t}}{2}\left(\begin{array}{cccc}
1 & \cos 2\left(\theta+\theta_{0}\right) & \sin 2\left(\theta+\theta_{0}\right) & 0 \\
\cos 2\left(\theta+\theta_{0}\right) & \cos ^{2} 2\left(\theta+\theta_{0}\right) & \sin 2\left(\theta+\theta_{0}\right) \cdot \cos 2\left(\theta+\theta_{0}\right) & 0 \\
\sin 2\left(\theta+\theta_{0}\right) & \sin 2\left(\theta+\theta_{0}\right) \cdot \cos 2\left(\theta+\theta_{0}\right) & \sin ^{2} 2\left(\theta+\theta_{0}\right) & 0 \\
0 & 0 & 0 & 0
\end{array}\right) \cdot\left(\begin{array}{l}
1 \\
1 \\
0 \\
0
\end{array}\right)
$$

And the intensity is given by $S_{0}^{\prime}$

$$
I(\theta)=\frac{t}{2}\left(1+\cos 2\left(\theta+\theta_{0}\right)\right)=\frac{t}{2}\left(1+\cos 2 \theta_{0} \cos 2 \theta-\sin 2 \theta_{0} \sin 2 \theta\right)
$$


Note that the intensity is a periodic function of the rotation angle $\theta$. The coefficients of the cosines and sinus terms can be determined by Fourier analysis. This is the basis of the synchronous detection. In fact, this method appears in many experimental data analysis like in phase shifting interferometers. Then, $\mathrm{N}$ intensity measures are taken at $\mathrm{N}$ equally spaced angles $\theta_{\mathrm{r}}=2 \pi(\mathrm{r}-1) / \mathrm{N} ; \mathrm{r}=1, \ldots, \mathrm{N}-1$. By multiplying the measured intensities by $\sin \left(2 \theta_{\mathrm{r}}\right)$ and adding the results one obtain

$$
2 \cdot \sum_{\mathrm{r}=1}^{\mathrm{N}} \mathrm{I}\left(\theta_{\mathrm{r}}\right) \cdot \sin 2 \theta_{\mathrm{r}}=\mathrm{t} \sum_{\mathrm{r}=1}^{\mathrm{N}} \sin 2 \theta_{\mathrm{r}}+\mathrm{t} \cdot \cos 2 \theta_{0} \cdot \sum_{\mathrm{r}=1}^{\mathrm{N}} \cos 2 \theta_{\mathrm{r}} \sin 2 \theta_{\mathrm{r}}-\mathrm{t} \cdot \sin 2 \theta_{0} \cdot \sum_{\mathrm{r}=1}^{\mathrm{N}} \sin 2 \theta_{\mathrm{r}} \sin 2 \theta_{\mathrm{r}}=-\mathrm{t} \cdot \sin 2 \theta_{0} \cdot \frac{\mathrm{N}}{2}
$$

In this last equality we have taken into account the orthogonal properties of the sinus and cosines (See Appendix). By multiplying the measured intensities by $\cos (2 \theta r)$, adding the results and again by taking into account the orthogonal properties of the sinusoidal functions we have:

$$
2 \cdot \sum_{\mathrm{r}=1}^{\mathrm{N}} \mathrm{I}\left(\theta_{\mathrm{r}}\right) \cdot \cos 2 \theta_{\mathrm{r}}=\mathrm{t} \sum_{\mathrm{r}=1}^{\mathrm{N}} \cos 2 \theta_{\mathrm{r}}+\mathrm{t} \cdot \cos 2 \theta_{0} \cdot \sum_{\mathrm{r}=1}^{\mathrm{N}} \cos 2 \theta_{\mathrm{r}} \cos 2 \theta_{\mathrm{r}}-\mathrm{t} \cdot \sin 2 \theta_{0} \cdot \sum_{\mathrm{r}=1}^{\mathrm{N}} \sin 2 \theta_{\mathrm{r}} \cos 2 \theta_{\mathrm{r}}=\mathrm{t} \cdot \cos 2 \theta_{0} \cdot \frac{\mathrm{N}}{2}
$$

Finally, the offset angle $\theta_{0}$ can be obtained as

$$
\theta_{0}=\frac{1}{2} \operatorname{arctg}\left(\frac{-\sum_{\mathrm{r}=1}^{\mathrm{N}} \mathrm{I}\left(\theta_{\mathrm{r}}\right) \cdot \sin 2 \theta_{\mathrm{r}}}{\sum_{\mathrm{r}=1}^{\mathrm{N}} \mathrm{I}\left(\theta_{\mathrm{r}}\right) \cdot \cos 2 \theta_{\mathrm{r}}}\right)
$$

Once this offset angle $\theta_{0}$ is measured, we can correct it when positioning the second polarizer. Figure 2(a) shows the plot of the measured intensity as a function of $\theta$. As the transmission axis was rotated an angle $\theta_{0}=27.775^{\circ}$ the sinusoidal function is shifted. Figure 2(b) shows the same plot once the offset is corrected when positioning the second polarizer.
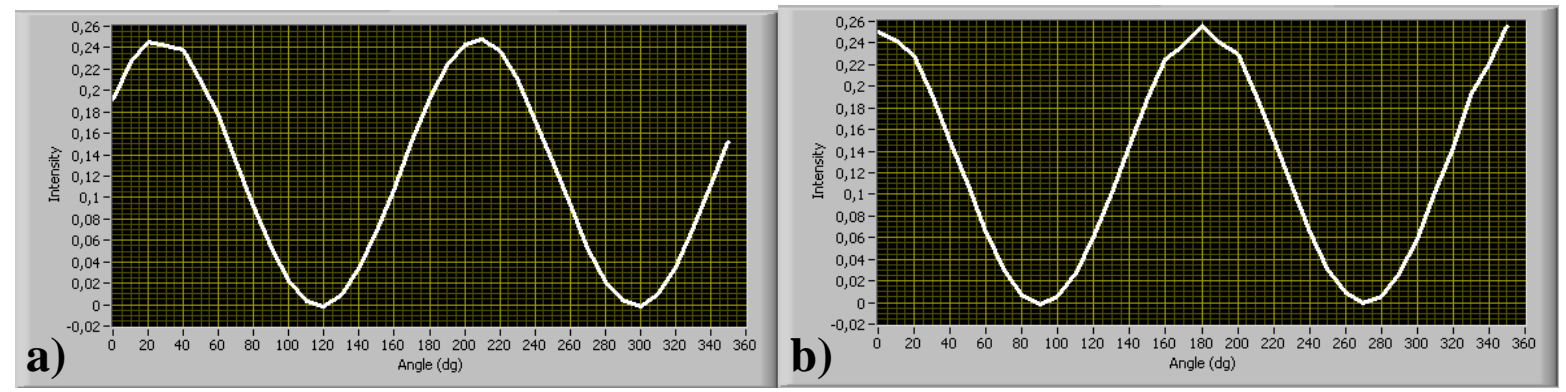

Figure 2. (a) Graph of the measured intensity I( $\theta$ ) from which the offset angle $\theta_{0}$ is determined. (b) The same graph once the offset has been corrected.

\subsection{Determination of the rotation and the phase-shift of a linear retarder placed between two polarizers}

Once we know the transmission axes of the polarizers, it is necessary to determine the fast axis of the retarder of retardance $\varphi$. This axis can be rotated an offset angle $\theta_{1}$ with respect to its rule. Let the two transmission axis of the polarizers be parallel, and the retarder rotated an angle $\theta$, that in fact will be $\theta+\theta_{1}$. The Stokes vector after the system is given by: 


$$
\left(\begin{array}{l}
\mathrm{S}_{0}^{\prime} \\
\mathrm{S}_{1}^{\prime} \\
\mathrm{S}_{2}^{\prime} \\
\mathrm{S}_{3}^{\prime}
\end{array}\right)=\mathrm{t} \cdot \mathrm{M}_{\mathrm{LP}}\left(0^{\circ}\right) \mathrm{M}_{\mathrm{WP}}\left(\varphi_{1}, \theta+\theta_{1}\right) \cdot\left(\begin{array}{l}
1 \\
1 \\
0 \\
0
\end{array}\right)=\frac{\mathrm{t}}{2}\left(\begin{array}{llll}
1 & 1 & 0 & 0 \\
1 & 1 & 0 & 0 \\
0 & 0 & 0 & 0 \\
0 & 0 & 0 & 0
\end{array}\right) \cdot\left(\begin{array}{c}
1 \\
\cos ^{2} 2\left(\theta+\theta_{1}\right)+\cos \varphi \sin ^{2} 2\left(\theta+\theta_{1}\right) \\
\left.\left.(1-\cos \varphi) \sin 2 \theta+\theta_{1}\right) \cos 2 \theta+\theta_{1}\right) \\
\left.\sin \varphi \sin 2 \theta+\theta_{1}\right)
\end{array}\right)
$$

Where the $M_{L P}$ and $M_{W P}$ matrices are given by Eq. (2) and Eq. (7) respectively. Then, the measured intensity is given by

$$
\mathrm{I}(\varphi, \theta)=\frac{\mathrm{t}}{4}\left(1+\left(\cos 4 \theta_{1} \cos 4 \theta_{1}-\sin 4 \theta_{1} \sin 4 \theta_{1}\right)(1-\cos \varphi)\right)
$$

Again this intensity is a periodic function of the rotation angle $\theta$. Then performing a Fourier analysis the offset angle can be determined. This intensity is measured for $N$ equally spaced angles $\theta_{\mathrm{r}}=2 \pi(\mathrm{r}-1) / \mathrm{N} ; \mathrm{r}=1, \ldots, \mathrm{N}-1$. By multiplying the measured intensities by $\sin \left(4 \theta_{\mathrm{r}}\right)$ or $\cos \left(4 \theta_{\mathrm{r}}\right)$ and adding the result one obtains

$$
\begin{aligned}
& 4 \cdot \sum_{\mathrm{r}=1}^{\mathrm{N}} \mathrm{I}\left(\varphi, \theta_{\mathrm{r}}\right) \sin \left(4 \theta_{\mathrm{r}}\right)=\ldots=-\mathrm{t}(1-\cos \varphi) \sin 4 \theta_{1} \frac{\mathrm{N}}{2} \\
& 4 \cdot \sum_{\mathrm{r}=1}^{\mathrm{N}} \mathrm{I}\left(\varphi, \theta_{\mathrm{r}}\right) \cos \left(4 \theta_{\mathrm{r}}\right)=\ldots=\mathrm{t}(1-\cos \varphi) \cos 4 \theta_{1} \frac{\mathrm{N}}{2}
\end{aligned}
$$

Then, the offset angle $\theta_{1}$ can be obtained as

$$
\theta_{1}=\frac{1}{4} \arctan \left(\frac{-\sum_{\mathrm{r}=1}^{\mathrm{N}} \mathrm{I}\left(\varphi, \theta_{\mathrm{r}}\right) \sin \left(4 \theta_{\mathrm{r}}\right)}{\sum_{\mathrm{r}=1}^{\mathrm{N}} \mathrm{I}\left(\varphi, \theta_{\mathrm{r}}\right) \cos \left(4 \theta_{\mathrm{r}}\right)}\right)
$$

Once this angle is know we can also obtain the phase shift of the retarder. By adding the intensities one obtain

$$
\begin{gathered}
4 \cdot \sum_{\mathrm{r}=1}^{\mathrm{N}} \mathrm{I}\left(\varphi, \theta_{\mathrm{r}}\right)=\mathrm{Nt} \\
\frac{4 \cdot \sum_{\mathrm{r}=1}^{\mathrm{N}} \mathrm{I}\left(\varphi, \theta_{\mathrm{r}}\right) \cos \left(4 \theta_{\mathrm{r}}\right)}{4 \cdot \sum_{\mathrm{r}=1}^{\mathrm{N}} \mathrm{I}\left(\varphi, \theta_{\mathrm{r}}\right)}=\frac{\mathrm{t}(1-\cos \varphi) \cos 4 \theta_{1} \frac{\mathrm{N}}{2}}{\mathrm{tN}}=\frac{1}{2} \cos 4 \theta_{1}(1-\cos \varphi)
\end{gathered}
$$

then

$$
\cos \varphi=1-\frac{2 \cdot \sum_{\mathrm{r}=1}^{\mathrm{N}} \mathrm{I}\left(\varphi, \theta_{\mathrm{r}}\right) \cos \left(4 \theta_{\mathrm{r}}\right)}{\cos 4 \theta_{1} \sum_{\mathrm{r}=1}^{\mathrm{N}} \mathrm{I}\left(\varphi, \theta_{\mathrm{r}}\right)}
$$


As an example of the calibration results, Figure 3 shows the plot of the measured intensity as a function of the waveplate rotation angle $\theta$, from which the waveplate offset angle $\theta_{1}$ is obtained. The sinusoidal function is consequence of the projection of the state of polarization exiting from the polarizer+waveplate system upon the fixed analyzer. The maximums correspond to the positions where the neutral lines of the waveplate are parallel to the polarizer transmission axis (it happens four times in a whole turn). On the other hand, the non null minimums correspond to the projection of circular polarized light. Then, from the shift of the first maximum from the $x=0$ position we obtain the $\theta_{1}$ angle and Eq. (21) gives us the phase-shift $\varphi$ value. The values corresponding to the sinusoidal function given in Fig. 3 are $\theta_{1}=29.546$ and $\varphi=90$ degrees.

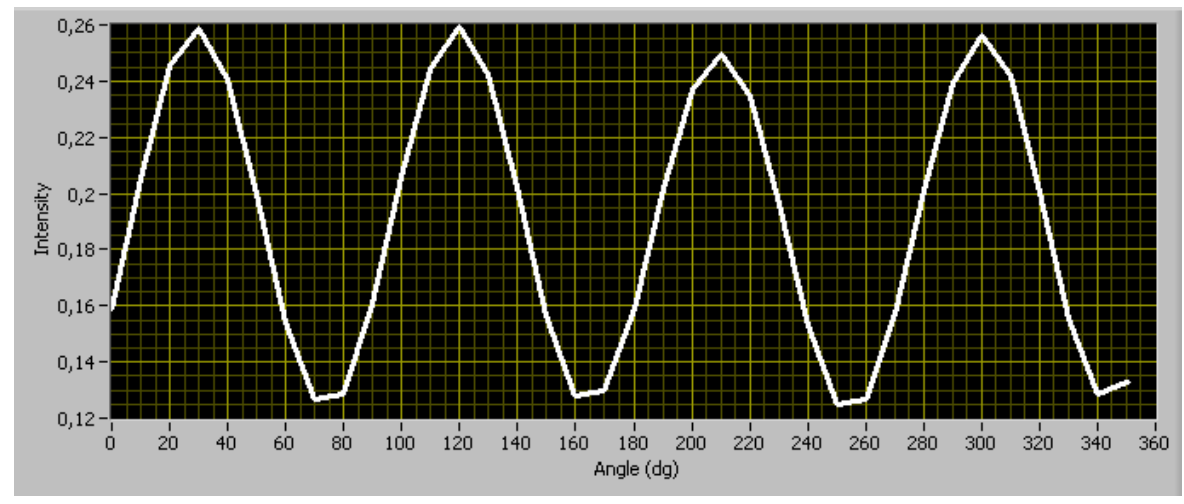

Figure 3. Graph of the measured intensity I $(\theta)$ from which the offset angle $\theta_{1}$ and phase shift $\varphi$ are determined

\section{STATE OF POLARIZATION AND POLARIZING SAMPLES CHARACTERIZATION}

In this section we detail the third unit of the experiment. Here, the students learn and practice the methodology which leads them to the obtaining of the SoP of light beams and of the Mueller matrix $M$ of polarizing elements. The physical interpretation of the results obtained is a key issue in the polarimetry training of the master students.

\subsection{Rotating linear retarder Stokes polarimeter}

Once the polarizers and the retarders are characterized we can build a Stokes polarimeter. The system is composed by a motorized rotating retarder $\left(\mathrm{WP}_{2}\right)$, a fixed polarizer at $0^{\circ}\left(\mathrm{LP}_{2}\right)$, and a photodetector (see Fig. 1). Let $\mathbf{S}$ be the Stokes vector to be measured. The stokes vector $\mathrm{S}^{\prime}$ after the retarder is

$$
\mathrm{S}^{\prime}=\mathrm{M}_{\mathrm{WPR}}(\varphi, \theta) \cdot \mathrm{S}=\mathrm{M}_{\mathrm{R}}(-\theta) \mathrm{M}_{\mathrm{WP}}\left(\varphi, 0^{\circ}\right) \mathrm{M}_{\mathrm{R}}(\theta) \mathrm{S}=\left(\begin{array}{c}
\mathrm{S}_{0} \\
\mathrm{~S}_{1}\left(\cos ^{2} 2 \theta+\cos \varphi \sin ^{2} 2 \theta\right)+\mathrm{S}_{2}(1-\cos \varphi) \cos 2 \theta \cdot \sin 2 \theta-\mathrm{S}_{3} \sin \varphi \sin 2 \theta \\
\mathrm{S}_{1}((1-\cos \varphi) \sin 2 \theta \cdot \cos 2 \theta)+\mathrm{S}_{2}\left(\sin ^{2} 2 \theta+\cos \varphi \cos ^{2} 2 \theta\right)+\mathrm{S}_{3}(\sin \varphi \cos 2 \theta) \\
\mathrm{S}_{1} \sin \varphi \sin 2 \theta-\mathrm{S}_{2} \sin \varphi \sin 2 \theta+\mathrm{S}_{3} \cos \varphi
\end{array}\right)
$$

And then the intensity after the polarizer is given by

$$
I(\varphi, \theta)=\frac{1}{2}\left[S_{0}+\frac{S_{1}}{2}(1+\cos \varphi)+\frac{S_{1}}{2}(1-\cos \varphi) \cos 4 \theta+\frac{S_{2}}{2}(1-\cos \varphi) \sin 4 \theta-S_{3} \sin \varphi \sin 2 \theta\right]
$$


In the particular case of a $\lambda / 4$ retarder $(\varphi=\pi / 2)$ the above expression is

$$
I\left(\frac{\pi}{2}, \theta\right)=\frac{1}{2}\left[S_{0}+\frac{S_{1}}{2}+\frac{S_{1}}{2} \cos 4 \theta+\frac{S_{2}}{2} \sin 4 \theta-S_{3} \sin 2 \theta\right]
$$

The measured intensities are periodical functions of the angle $\theta$, and contain several sinusoidal functions whose coefficients depend on the Stokes parameters. A Fourier analysis permits us to obtain these coefficients. To this end $\mathrm{N}$ measures at equally spaced angles of the $\mathrm{WP}_{2} \theta_{r}=2 \pi(r-1) / N ; r=1, \ldots, N-1$ are taken and multiplied by the corresponding value of the sinusoidals. Then the following expressions are obtained:

$$
\left\{\begin{aligned}
2 \cdot \sum_{\mathrm{r}=1}^{\mathrm{N}} \mathrm{I}\left(\varphi, \theta_{\mathrm{r}}\right) & =\sum_{\mathrm{r}=1}^{\mathrm{N}}\left(\mathrm{S}_{0}+\frac{\mathrm{S}_{1}}{2}(1+\cos \varphi)\right)=\mathrm{N}\left(\mathrm{S}_{0}+\frac{\mathrm{S}_{1}}{2}(1+\cos \varphi)\right) \\
2 \cdot \sum_{\mathrm{r}=1}^{\mathrm{N}} \mathrm{I}\left(\varphi, \theta_{\mathrm{r}}\right) \cdot \sin 2 \theta_{\mathrm{r}} & =-\frac{\mathrm{N}}{2} \cdot \mathrm{S}_{3} \sin \varphi \\
2 \cdot \sum_{\mathrm{r}=1}^{\mathrm{N}} \mathrm{I}\left(\varphi, \theta_{\mathrm{r}}\right) \cdot \sin 4 \theta_{\mathrm{r}} & =\frac{\mathrm{N}}{4} \mathrm{~S}_{2}(1-\cos \varphi) \\
2 \cdot \sum_{\mathrm{r}=1}^{\mathrm{N}} \mathrm{I}\left(\varphi, \theta_{\mathrm{r}}\right) \cdot \cos 4 \theta_{\mathrm{r}} & =\frac{\mathrm{N}}{4} \mathrm{~S}_{1}(1-\cos \varphi)
\end{aligned}\right.
$$

And then the Stokes vector can be obtained as

$$
\left(\begin{array}{l}
\mathrm{S}_{0} \\
\mathrm{~S}_{1} \\
\mathrm{~S}_{2} \\
\mathrm{~S}_{3}
\end{array}\right)=\frac{1}{\mathrm{~N}}\left(\begin{array}{c}
\left(2 \cdot \sum_{\mathrm{r}=1}^{\mathrm{N}} \mathrm{I}\left(\varphi, \theta_{\mathrm{r}}\right)-4 \frac{1+\cos \varphi}{1-\cos \varphi} \sum_{\mathrm{r}=1}^{\mathrm{N}} \mathrm{I}\left(\varphi, \theta_{\mathrm{r}}\right) \cdot \cos 4 \theta_{\mathrm{r}}\right) \\
\frac{8}{1-\cos \varphi} \sum_{\mathrm{r}=1}^{\mathrm{N}} \mathrm{I}\left(\varphi, \theta_{\mathrm{r}}\right) \cdot \cos 4 \theta_{\mathrm{r}} \\
\frac{8}{1-\cos \varphi} \sum_{\mathrm{r}=1}^{\mathrm{N}} \mathrm{I}\left(\varphi, \theta_{\mathrm{r}}\right) \cdot \sin 4 \theta_{\mathrm{r}} \\
\frac{-4}{\sin \varphi} \sum_{\mathrm{r}=1}^{\mathrm{N}} \mathrm{I}\left(\varphi, \theta_{\mathrm{r}}\right) \cdot \sin 2 \theta_{\mathrm{r}}
\end{array}\right)
$$

In the laboratory, the students measure different SoPs: linear states of polarization with different orientation, right-handed and left-handed circular polarization and diverse elliptical states of polarization. These SoPs are generated by themselves by properly setting configurations of external quarter-waveplates and polarimeteres. Then, they interpret the experimental Stokes parameters obtained by using the polarization state detector (Fig. 1) and Eq. (26). Some polarimetric concepts, as unpolarized light, azimuth angle ( $\alpha$ ) or ellipticity angle $(\varepsilon)^{9}$ are revised by taking advantage of the obtained results. As an example, Table 2 shows some students measurements corresponding to the detection of linear polarized light at $0^{\circ}$ of the laboratory vertical, righthanded circular polarized light and a specific elliptical polarized light. The ellipses of polarization of the SoPs shown in Table 2 are plotted in Fig. 4. In the Fig. 4(c), the azimuth and ellipticity angles are also represented. 


\begin{tabular}{|c|c|c|c|}
\cline { 2 - 4 } \multicolumn{1}{c|}{} & Linear polarization $\left(0^{\circ}\right)$ & Right-handed polarization & Elliptical polarization \\
\hline \multirow{3}{*}{$\begin{array}{c}\text { Theoretical } \\
\text { values }\end{array}$} & 1 & 1 & 1 \\
\cline { 2 - 4 } & 1 & 0 & 0.587 \\
\cline { 2 - 4 } & 0 & 0 & -0.492 \\
\hline \multirow{3}{*}{$\begin{array}{c}\text { Experimental } \\
\text { measurement }\end{array}$} & 1 & 1 & -0.643 \\
\cline { 2 - 4 } & 0.999 & 1 & 1 \\
\cline { 2 - 4 } & $2.013 \cdot 10^{-3}$ & -0.063 & 0.578 \\
\cline { 2 - 4 } & $2.443 \cdot 10^{-3}$ & $0.928 \cdot 10^{-3}$ & -0.521 \\
\hline
\end{tabular}

Table 2. Some Stokes parameters obtained by master students.
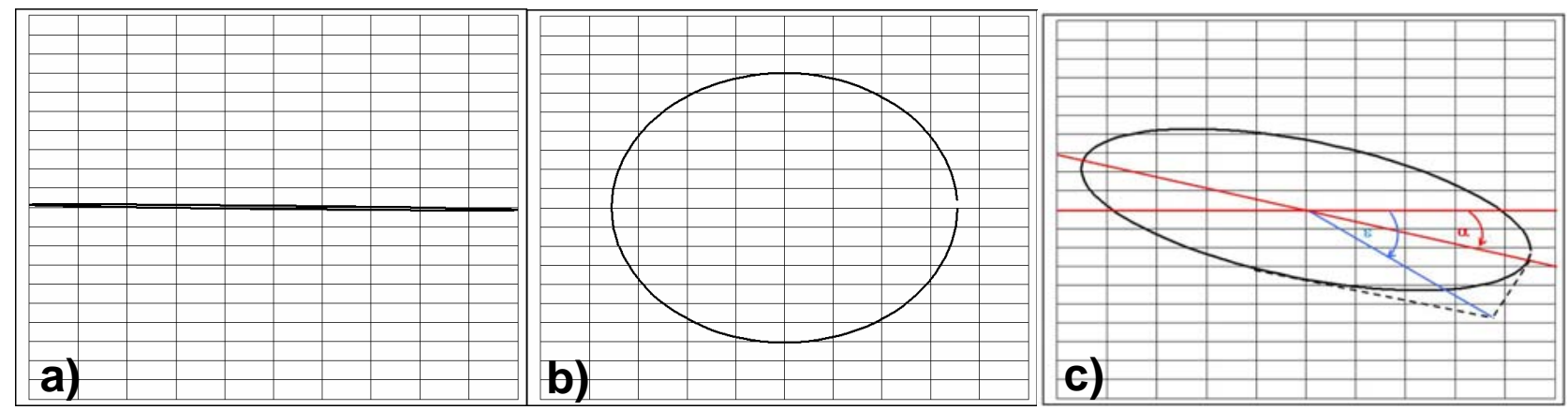

Figure 4. Ellipse of polarization of the SoP shown in Table 1.

\subsection{Rotating linear retarders Mueller polarimeter}

The Mueller polarimeter contains a Polarization State Generator (PSG) composed by a linear polarizer $\mathrm{LP}_{1}$ at $0^{\circ}$ and a rotating linear retarder $\mathrm{WP}_{1}$ with phase shift $\varphi^{1}$ and a Polarization State Detector (PSD) like the described in the previous section. When the linear retarder of the PSG is at a rotation $\theta^{\mathrm{r}}$, the generated SoP is given by

$$
\mathrm{Si}^{\mathrm{r}}=\left(\begin{array}{c}
1 \\
\frac{1}{2}\left(1+\cos \varphi^{1}\right)+\frac{1}{2}\left(1-\cos \varphi^{1}\right) \cos 4 \theta^{\mathrm{r}} \\
\frac{1}{2}\left(1-\cos \varphi^{1}\right) \sin 4 \theta^{\mathrm{r}} \\
\sin \varphi^{1} \sin 2 \theta^{\mathrm{r}}
\end{array}\right)
$$

This input SoP passes through the polarizing device $M$ that we want to characterize and the output SoP is given by

$$
\mathrm{So}^{\mathrm{r}}=\left(\begin{array}{cccc}
\mathrm{m}_{00} & \mathrm{~m}_{01} & \mathrm{~m}_{02} & \mathrm{~m}_{03} \\
\mathrm{~m}_{10} & \mathrm{~m}_{11} & \mathrm{~m}_{12} & \mathrm{~m}_{13} \\
\mathrm{~m}_{20} & \mathrm{~m}_{21} & \mathrm{~m}_{22} & \mathrm{~m}_{23} \\
\mathrm{~m}_{30} & \mathrm{~m}_{31} & \mathrm{~m}_{32} & \mathrm{~m}_{33}
\end{array}\right) \cdot \mathrm{Si}^{\mathrm{r}}
$$


The k component of this output SoP is given by

$$
\begin{aligned}
\mathrm{So}_{\mathrm{k}}^{\mathrm{r}} & =\mathrm{m}_{\mathrm{k} 0}+\mathrm{m}_{\mathrm{k} 1} \frac{1}{2}\left(\left(1+\cos \varphi^{1}\right)+\left(1-\cos \varphi^{1}\right) \cos 4 \theta^{\mathrm{r}}\right)+\mathrm{m}_{\mathrm{k} 2} \frac{1}{2}\left(1-\cos \varphi^{1}\right) \sin 4 \theta^{\mathrm{r}}+\mathrm{m}_{\mathrm{k} 3} \sin \varphi^{1} \sin 2 \theta^{\mathrm{r}} \\
& =\mathrm{m}_{\mathrm{k} 0}+\frac{1}{2}\left(1+\cos \varphi^{1}\right) \mathrm{m}_{\mathrm{k} 1}+\frac{1}{2}\left(1-\cos \varphi^{1}\right) \mathrm{m}_{\mathrm{k} 1} \cos 4 \theta^{\mathrm{r}}+\mathrm{m}_{\mathrm{k} 2} \frac{1}{2}\left(1-\cos \varphi^{1}\right) \sin 4 \theta^{\mathrm{r}}+\mathrm{m}_{\mathrm{k} 3} \sin \varphi^{1} \sin 2 \theta^{\mathrm{r}}
\end{aligned}
$$

This output SoP can be measured by the PSD. Then, if $\mathrm{N}$ equally spaced rotations are used in the PSG, the coefficients of the matrix can be calculated as:

$$
\begin{gathered}
\sum_{r=1}^{N} S o_{K}^{r} \cdot \cos 4 \theta^{r}=\frac{N}{4}\left(1-\cos \varphi^{1}\right) m_{k 1} \\
\sum_{r=1}^{N} S o_{K}^{r} \cdot \sin 4 \theta^{r}=\frac{N}{4}\left(1-\cos \varphi^{1}\right) m_{k 2} \\
\sum_{r=1}^{N} S o_{K}^{r} \cdot \sin 2 \theta^{r}=\frac{N}{2} \sin \varphi^{1} m_{k 3} \\
\sum_{r=1}^{N} S o_{K}^{r}=N m_{k 0}+\frac{N}{2}\left(1+\cos \varphi^{1}\right) m_{k 1}=N m_{k 0}+2 \frac{\left(1+\cos \varphi^{1}\right)}{\left(1-\cos \varphi^{1}\right)} \sum_{r=1}^{N} S_{K}^{r} \cdot \cos 4 \theta^{r}
\end{gathered}
$$

Then the Mueller matrix of the device is

$$
M=\frac{1}{N}\left(\begin{array}{llll}
\sum_{r=1}^{N} S_{0}^{r}-2 \frac{1+\cos \varphi^{1}}{1-\cos \varphi^{1}} \sum_{r=1}^{N} S_{0}^{r} \cdot \cos 4 \theta^{r} & \frac{4}{1-\cos \varphi^{1}} \sum_{r=1}^{N} S_{0}^{r} \cdot \cos 4 \theta^{r} & \frac{4}{1-\cos \varphi^{1}} \sum_{r=1}^{N} S_{0}^{r} \cdot \sin 4 \theta^{r} & \frac{2}{\sin \varphi^{1}} \sum_{r=1}^{N} S_{0}^{r} \cdot \sin 2 \theta^{r} \\
\sum_{r=1}^{N} S_{1}^{r}-2 \frac{1+\cos \varphi^{1}}{1-\cos \varphi^{1}} \sum_{r=1}^{N} S_{1}^{r} \cdot \cos 4 \theta^{r} & \frac{4}{1-\cos \varphi^{1}} \sum_{r=1}^{N} S_{1}^{r} \cdot \cos 4 \theta^{r} & \frac{4}{1-\cos \varphi^{1}} \sum_{r=1}^{N} S_{1}^{r} \cdot \sin 4 \theta^{r} & \frac{2}{\sin \varphi^{1}} \sum_{r=1}^{N} S_{1}^{r} \cdot \sin 2 \theta^{r} \\
\sum_{r=1}^{N} S_{2}^{r}-2 \frac{1+\cos \varphi^{1}}{1-\cos \varphi^{1}} \sum_{r=1}^{N} S_{2}^{r} \cdot \cos 4 \theta^{r} & \frac{4}{1-\cos \varphi^{1}} \sum_{r=1}^{N} S_{2}^{r} \cdot \cos 4 \theta^{r} & \frac{4}{1-\cos \varphi^{1}} \sum_{r=1}^{N} S_{2}^{r} \cdot \sin 4 \theta^{r} & \frac{2}{\sin \varphi^{1}} \sum_{r=1}^{N} S_{2}^{r} \cdot \sin 2 \theta^{r} \\
\sum_{r=1}^{N} S_{3}^{r}-2 \frac{1+\cos \varphi^{1}}{1-\cos \varphi^{1}} \sum_{r=1}^{N} S_{3}^{r} \cdot \cos 4 \theta^{r} & \frac{4}{1-\cos \varphi^{1}} \sum_{r=1}^{N} S_{3}^{r} \cdot \cos 4 \theta^{r} & \frac{4}{1-\cos \varphi^{1}} \sum_{r=1}^{N} S_{3}^{r} \cdot \sin 4 \theta^{r} & \frac{2}{\sin \varphi^{1}} \sum_{r=1}^{N} S_{3}^{r} \cdot \sin 2 \theta^{r}
\end{array}\right)
$$

In this case, master students measure different polarizing elements with the aim of understand the physical meaning of the Mueller coefficients. The experimental obtaining of the Mueller matrices of the elements tested are accomplished by the properly combination of the polarization state generator and the polarization state detector plotted in Fig. 1 and by following Eq. (31). In particular, among others, they obtain the Mueller matrix of polarizers with different orientation, of linear retarders of different retardance, of adhesive tapes with different thickness and of a Faraday rotator. They also have the chance of characterize the Mueller matrix of any polarizing element that they bring to the laboratory. Some polarimetric concepts as diattenuation, retardance, polarizance or depolarization ${ }^{8}$ are revised by taking advantage of the obtained results. As an example, Table 3 shows the obtained Mueller matrix for an adhesive tape strip of a given thickness (Table $3(a)$ ) and for two crossed strips of adhesive tapes of the same thickness (Table 3(b)). Note that in both cases the diattenuation vector (the three last coefficients of the first row) and the polarizance vector (the three last coefficients of the first column) are almost null, and as a consequence we are obtaining the Mueller matrix of a retarder of a given orientation and retardance. However, when using two crossed strips the phase added by one strip to one component of the electric field is subtracted by the other strip, leading to the identity matrix. It can be observed by normalizing the matrix shown in Table $3(b)$ by the $m_{00}$ Mueller matrix coefficient (the irradiance exiting from the polarizing sample). In other words, the characterization of two identical crossed strips of adhesive tape is like performing a characterization without any polarizing sample. 


$\left.\begin{array}{|cccc}0.253 & 1.01 \cdot 10^{-2} & -2.50 \cdot 10^{-2} & 2.90 \cdot 10^{-2} \\ 5.77 \cdot 10^{-3} & 0.231 & 0.147 & -5.57 \cdot 10^{-2} \\ -3.75 \cdot 10^{-2} & 0.133 & -0.120 & 0.206 \\ -4.47 \cdot 10^{-2} & 8.70 \cdot 10^{-2} & -0.205 & -0.146\end{array}\right) \mid \quad$ b) $\quad\left(\begin{array}{ccccc|}0.255 & -0.016 & 0.040 & 0.003 \\ -0.021 & 0.271 & 0.027 & 0.005 \\ 0.052 & -0.025 & 0.273 & 0.010 \\ 0.002 & -0.010 & -0.005 & 0.239\end{array}\right)$

Table 3. Mueller matrices of an adhesive tape: (a) one strip; (b) two strips crossed.

\section{SUMMARY}

Polarimetric techniques are a very useful tool in many research fields as optics, medicine or biology. A polarimeter is the basic scientific instrument used to make polarimetric measurements, leading to the characterization of the state of polarization of light beams or to the obtaining of some polarization information of polarizing samples. Therefore, the spreading of the basic knowledge of this topic becomes interesting for many professionals and a master studies is an excellent environment to this aim.

By participating in a mandatory laboratory subject (Laboratory of Optics, LO) of a Master degree in Photonics we are conducting an experiment on polarization. The main structure of the experiment has been built around of a polarimeter set-up that allows us the obtaining of the complete description of any polarizing element tested. The polarimetric experiment is subdivided in three different units which lead to specific educational trainings. In this paper, a detailed explanation of the experiment is provided.

Basically, the first unit gives students the fundamental background required for the understanding of the experiment, being in particular the basis of the Mueller-Stokes formalism. The second unit allows us to lead them into a set-up calibration performance based on the synchronous detection and the M-S formalism. This knowledge is a first step into the M-S application and provides students with a calibration procedure able to optimize the performance of any optical system involving polarizing elements. Finally, in the third unit the students use the polarimeter to detect different states of polarization and to characterize diverse polarizing elements. By taking advantage of this third unit of the experiment, concepts as unpolarized light, ellipticity, azimuth angle, despolarization, retardance or diattenuation are revised. The third unit is an essential part of the experiment, leading students to important issues as the manipulation of the set-up, to the theory reinforce by using different practical examples, and to the polarimetric analysis of the obtained results. Additionally, the students have to deliver a mandatory report and to perform an oral presentation. This fact adds complementary information to the scientific training of the students. In this sense, the developing of the reports help students to achieve a further internalization of the experiment done, show them the benefits of the team work and help them to improve their communicative skills.

Finally, we want to emphasize that the mandatory laboratory subject is structured with the aim of provide students with a minimum knowledge level. However, as a function of the specific background of every student in polarimetry, they are trained with a different degree of complexity. For instants, advanced students are taught with a higher degree in depth when performing the polarimetric data analysis, they are able to work with the LabVIEW software font code, to perform simulations in MATLAB or C++ of the estimated error associated to the measurements, among others. 


\section{APPENDIX}

The equally spaced sampled sinusoidal functions fulfill the following properties:

$$
\begin{gathered}
\frac{2}{\mathrm{~N}} \sum_{\mathrm{r}=1}^{\mathrm{N}} \sin \frac{2 \pi \mathrm{ri}}{\mathrm{N}} \cdot \sin \frac{2 \pi \mathrm{rj}}{\mathrm{N}}=\frac{2}{\mathrm{~N}} \sum_{\mathrm{r}=1}^{\mathrm{N}} \cos \frac{2 \pi \mathrm{ri}}{\mathrm{N}} \cdot \cos \frac{2 \pi \mathrm{rj}}{\mathrm{N}}=\delta_{\mathrm{ij}} \\
\frac{2}{\mathrm{~N}} \sum_{\mathrm{r}=1}^{\mathrm{N}} \sin \frac{2 \pi \mathrm{ri}}{\mathrm{N}} \cdot \cos \frac{2 \pi \mathrm{rj}}{\mathrm{N}}=0 \\
\sum_{\mathrm{r}=1}^{\mathrm{N}} \sin \frac{2 \pi \mathrm{ri}}{\mathrm{N}}=\sum_{\mathrm{r}=1}^{\mathrm{N}} \cos \frac{2 \pi \mathrm{ri}}{\mathrm{N}}=0
\end{gathered}
$$

\section{ACKNOWLEDGMENTS}

The authors acknowledge financial support from the Spanish Ministerio de Educación y Ciencia (grant FIS2006-13037-C02-01).

\section{REFERENCES}

[1] M. Anastasiadou, A. De Martino, D. Clement, F. Liège, B. Laude-Boulesteix, N. Quang, J. Dreyfuss, B. Huynh, A. Nazac, L. Schwartz, and H. A. S. Cohen, "Polarimetric imaging for the diagnosis of cervical cancer", Phys. Stat. Sol., 5 (5), (2008).

[2] K. M. Twietmeyer, R. A. Chipman, A. E. Elsner, Y. Zhao, and D. VanNasdale, "Mueller matrix retinal imager with optimized polarization conditions," Opt. Express, 16 (26), (2008).

[3] L. J. November and L.M. Wilkins, "The Liquid Crystal Polarimeter for solid-state imaging of solar vector magnetic fields" Proc. SPIE 2265, 210-221 (1992).

[4] A. Márquez, I. Moreno, C. Iemmi, A. Lizana, J. Campos and J.M. Yzuel, "Mueller-Stokes characterization and optimization of a liquid crystal on silicon display showing depolarization" Opt. Express, 16, 1669-1685 (2008).

[5] S. Firdous M. Ikram "Stokes Polarimetry for the Characterization of Bio-Materials using Liquid Crystal Variable Retarders" Proc. of the SPIE-OSA Biomedical Optics 6632, 66320F-1 66320F-13 (2007).

[6] R. A. Chipman in [Handbook of Optics], 2nd ed., McGraw-Hill, New York, Chapter 22 (1995).

[7] R.M.A. Azzam and N.M. Bashara, [Ellipsometry and Polarized Light], North-Holland, Oxford, (1977).

[8] D. Goldstein, [Polarized Light], $2^{\text {nd }}$ ed., Marcel Dekker, New York, (2003).

[9] J. Casas, [Óptica], $7^{\text {th }}$ ed., Librería Pons, Zaragoza, (1994). 